\title{
Infinite generation of non-cocompact lattices on right-angled buildings
}

\author{
ANNE THOMAS \\ KEVIN WORTMAN
}

\begin{abstract}
Let $\Gamma$ be a non-cocompact lattice on a locally finite regular right-angled building $X$. We prove that if $\Gamma$ has a strict fundamental domain then $\Gamma$ is not finitely generated. We use the separation properties of subcomplexes of $X$ called tree-walls.
\end{abstract}

20E42, 20F05; 20F55, 57M07, 51E24

Tree lattices have been well-studied (see Bass and Lubotsky [2]). Less understood are lattices on higher-dimensional CAT $(0)$ complexes. In this paper, we consider lattices on $X$ a locally finite, regular right-angled building (see Davis [5] and Section 1 below). Examples of such $X$ include products of locally finite regular or biregular trees, or Bourdon's building $I_{p, q}$ [3], which has apartments hyperbolic planes tesselated by right-angled $p$-gons and all vertex links the complete bipartite graph $K_{q, q}$.

Let $G$ be a closed, cocompact group of type-preserving automorphisms of $X$, equipped with the compact-open topology, and let $\Gamma$ be a lattice in $G$. That is, $\Gamma$ is discrete and the series $\sum\left|\operatorname{Stab}_{\Gamma}(\phi)\right|^{-1}$ converges, where the sum is over the set of chambers $\phi$ of a fundamental domain for $\Gamma$. The lattice $\Gamma$ is cocompact in $G$ if and only if the quotient $\Gamma \backslash X$ is compact.

If there is a subcomplex $Y \subset X$ containing exactly one point from each $\Gamma$-orbit on $X$, then $Y$ is called a strict fundamental domain for $\Gamma$. Equivalently, $\Gamma$ has a strict fundamental domain if $\Gamma \backslash X$ may be embedded in $X$.

Any cocompact lattice in $G$ is finitely generated. We prove:

Theorem 1 Let $\Gamma$ be a non-cocompact lattice in $G$. If $\Gamma$ has a strict fundamental domain, then $\Gamma$ is not finitely generated.

We note that Theorem 1 contrasts with the finite generation of lattices on many buildings whose chambers are simplices. Results of, for example, Ballmann and Świątkowski [1], Dymara and Januszkiewicz [6] and Zuk [13], establish that all lattices on many such buildings have Kazhdan's Property (T). Hence by a well-known result due to Kazhdan [8], these lattices are finitely generated. 
Our proof of Theorem 1, in Section 3 below, uses the separation properties of subcomplexes of $X$ which we call tree-walls. These generalize the tree-walls (in French, arbre-murs) of $I_{p, q}$, which were introduced by Bourdon in [3]. We define tree-walls and establish their properties in Section 2 below.

The following examples of non-cocompact lattices on right-angled buildings are known to us.

(1) For $i=1,2$, let $G_{i}$ be a rank one Lie group over a nonarchimedean locally compact field whose Bruhat-Tits building is the locally finite regular or biregular tree $T_{i}$. Then any irreducible lattice in $G=G_{1} \times G_{2}$ is finitely generated, by Raghunathan [10]. Hence by Theorem 1 above, such lattices on $X=T_{1} \times T_{2}$ cannot have strict fundamental domain.

(2) Let $\Lambda$ be a minimal Kac-Moody group over a finite field $\mathbb{F}_{q}$ with right-angled Weyl group $W$. Then $\Lambda$ has locally finite, regular right-angled twin buildings $X_{+} \cong X_{-}$, and $\Lambda$ acts diagonally on the product $X_{+} \times X_{-}$. For $q$ large enough:

(a) By Theorem 0.2 of Carbone and Garland [4] or Theorem 1(i) of Rémy [11], the stabilizer in $\Lambda$ of a point in $X_{-}$is a non-cocompact lattice in $\operatorname{Aut}\left(X_{+}\right)$. Any such lattice is contained in a negative maximal spherical parabolic subgroup of $\Lambda$, which has strict fundamental domain a sector in $X_{+}$, and so any such lattice has strict fundamental domain.

(b) By Theorem 1(ii) of Rémy [11], the group $\Lambda$ is itself a non-cocompact lattice in $\operatorname{Aut}\left(X_{+}\right) \times \operatorname{Aut}\left(X_{-}\right)$. Since $\Lambda$ is finitely generated, Theorem 1 above implies that $\Lambda$ does not have strict fundamental domain in $X=X_{+} \times X_{-}$.

(c) By Section 7.3 of Gramlich, Horn and Mühlherr [7], the fixed set $G_{\theta}$ of certain involutions $\theta$ of $\Lambda$ is a lattice in $\operatorname{Aut}\left(X_{+}\right)$, which is sometimes cocompact and sometimes non-cocompact. Moreover, by [7, Remark 7.13], there exists $\theta$ such that $G_{\theta}$ is not finitely generated.

(3) In [12], the first author constructed a functor from graphs of groups to complexes of groups, which extends the corresponding tree lattice to a lattice in $\operatorname{Aut}(X)$ where $X$ is a regular right-angled building. The resulting lattice in $\operatorname{Aut}(X)$ has strict fundamental domain if and only if the original tree lattice has strict fundamental domain.

Acknowledgements The first author was supported in part by NSF grant number DMS-0805206 and in part by EPSRC grant number EP/D073626/2, and is currently supported by ARC grant number DP110100440. The second author is supported in part by NSF grant number DMS-0905891. We thank Martin Bridson and Pierre-Emmanuel Caprace for helpful conversations. 


\section{Right-angled buildings}

In this section we recall the basic definitions and some examples for right-angled buildings. We mostly follow Davis [5], in particular Section 12.2 and Example 18.1.10. See also Kubena and Thomas [9, Sections 1.2-1.4].

Let $(W, S)$ be a right-angled Coxeter system. That is,

$$
W=\left\langle S \mid(s t)^{m_{s t}}=1\right\rangle
$$

where $m_{s s}=1$ for all $s \in S$, and $m_{s t} \in\{2, \infty\}$ for all $s, t \in S$ with $s \neq t$. We will discuss the following examples:

- $W_{1}=\left\langle s, t \mid s^{2}=t^{2}=1\right\rangle \cong D_{\infty}$, the infinite dihedral group;

- $W_{2}=\left\langle r, s, t \mid r^{2}=s^{2}=t^{2}=(r s)^{2}=1\right\rangle \cong\left(C_{2} \times C_{2}\right) * C_{2}$, where $C_{2}$ is the cyclic group of order 2 ; and

- The Coxeter group $W_{3}$ generated by the set of reflections $S$ in the sides of a right-angled hyperbolic $p$-gon, $p \geq 5$. That is,

$$
W_{3}=\left\langle s_{1}, \ldots, s_{p} \mid s_{i}^{2}=\left(s_{i} s_{i+1}\right)^{2}=1\right\rangle
$$

with cyclic indexing.

Fix $\left(q_{s}\right)_{s \in S}$ a family of integers with $q_{s} \geq 2$. Given any family of groups $\left(H_{s}\right)_{s \in S}$ with $\left|H_{S}\right|=q_{s}$, let $H$ be the quotient of the free product of the $\left(H_{S}\right)_{s \in S}$ by the normal subgroup generated by the commutators $\left\{\left[h_{s}, h_{t}\right]: h_{s} \in H_{s}, h_{t} \in H_{t}, m_{s t}=2\right\}$.

Now let $X$ be the piecewise Euclidean CAT(0) geometric realization of the chamber system $\Phi=\Phi\left(H,\{1\},\left(H_{S}\right)_{s \in S}\right)$. Then $X$ is a locally finite, regular right-angled building, with chamber set $\mathrm{Ch}(X)$ in bijection with the elements of the group $H$. Let $\delta_{W}: \mathrm{Ch}(X) \times \mathrm{Ch}(X) \rightarrow W$ be the $W$-valued distance function and let $l_{S}: W \rightarrow \mathbb{N}$ be word length with respect to the generating set $S$. Denote by $d_{W}: \operatorname{Ch}(X) \times \operatorname{Ch}(X) \rightarrow \mathbb{N}$ the gallery distance $l_{S} \circ \delta_{W}$. That is, for two chambers $\phi$ and $\phi^{\prime}$ of $X, d_{W}\left(\phi, \phi^{\prime}\right)$ is the length of a minimal gallery from $\phi$ to $\phi^{\prime}$.

Suppose that $\phi$ and $\phi^{\prime}$ are $s$-adjacent chambers, for some $s \in S$. That is, $\delta_{W}\left(\phi, \phi^{\prime}\right)=s$. The intersection $\phi \cap \phi^{\prime}$ is called an $s$-panel. By definition, since $X$ is regular, each $s$-panel is contained in $q_{s}$ distinct chambers. For distinct $s, t \in S$, the $s$-panel and $t$-panel of any chamber $\phi$ of $X$ have nonempty intersection if and only if $m_{s t}=2$. Each $s$-panel of $X$ is reduced to a vertex if and only if $m_{s t}=\infty$ for all $t \in S-\{s\}$. 
For the examples $W_{1}, W_{2}$, and $W_{3}$ above, respectively:

- The building $X_{1}$ is a tree with each chamber an edge, each $s$-panel a vertex of valence $q_{s}$, and each $t$-panel a vertex of valence $q_{t}$. That is, $X_{1}$ is the $\left(q_{s}, q_{t}\right)$-biregular tree. The apartments of $X_{1}$ are bi-infinite rays in this tree.

- The building $X_{2}$ has chambers and apartments as shown in Figure 1 below. The $r$ - and $s$-panels are 1-dimensional and the $t$-panels are vertices.
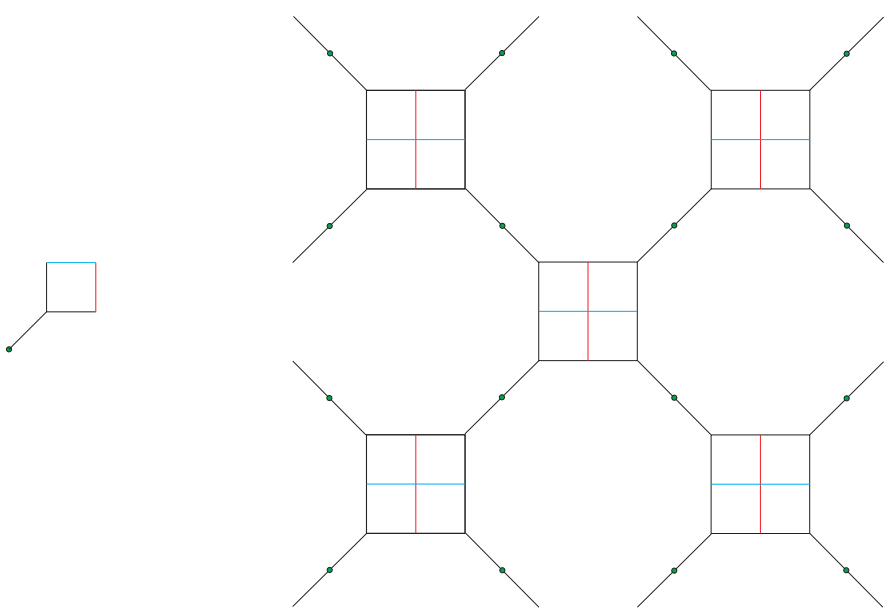

Figure 1: A chamber (on the left) and part of an apartment (on the right) for the building $X_{2}$.

- The building $X_{3}$ has chambers $p$-gons and $s$-panels the edges of these $p$-gons. If $q_{s}=q \geq 2$ for all $s \in S$, then each $s$-panel is contained in $q$ chambers, and $X_{3}$, equipped with the obvious piecewise hyperbolic metric, is Bourdon's building $I_{p, q}$.

\section{Tree-walls}

We now generalize the notion of tree-wall due to Bourdon [3]. We will use basic facts about buildings, found in, for example, Davis [5]. Our main results concerning tree-walls are Corollary 3 below, which describes three possibilities for tree-walls, and Proposition 6 below, which generalizes the separation property 2.4.A(ii) of [3].

Let $X$ be as in Section 1 above and let $s \in S$. As in [3, Section 2.4.A], we define two $s$-panels of $X$ to be equivalent if they are contained in a common wall of type $s$ in some apartment of $X$. A tree-wall of type $s$ is then an equivalence class under this 
relation. We note that in order for walls and thus tree-walls to have a well-defined type, it is necessary only that all finite $m_{s t}$, for $s \neq t$, be even. Tree-walls could thus be defined for buildings of type any even Coxeter system, and they would have properties similar to those below. We will however only explicitly consider the right-angled case.

Let $\mathcal{T}$ be a tree-wall of $X$, of type $s$. We define a chamber $\phi$ of $X$ to be epicormic at $\mathcal{T}$ if the $s$-panel of $\phi$ is contained in $\mathcal{T}$, and we say that a gallery $\alpha=\left(\phi_{0}, \ldots, \phi_{n}\right)$ crosses $\mathcal{T}$ if, for some $0 \leq i<n$, the chambers $\phi_{i}$ and $\phi_{i+1}$ are epicormic at $\mathcal{T}$.

By the definition of tree-wall, if $\phi \in \operatorname{Ch}(X)$ is epicormic at $\mathcal{T}$ and $\phi^{\prime} \in \operatorname{Ch}(X)$ is $t$-adjacent to $\phi$ with $t \neq s$, then $\phi^{\prime}$ is epicormic at $\mathcal{T}$ if and only if $m_{s t}=2$. Let $s^{\perp}:=\left\{t \in S \mid m_{s t}=2\right\}$ and denote by $\left\langle s^{\perp}\right\rangle$ the subgroup of $W$ generated by the elements of $s^{\perp}$. If $s^{\perp}$ is empty then by convention, $\left\langle s^{\perp}\right\rangle$ is trivial. For the examples in Section 1 above:

- in $W_{1}$, both $\left\langle s^{\perp}\right\rangle$ and $\left\langle t^{\perp}\right\rangle$ are trivial;

- in $W_{2},\left\langle r^{\perp}\right\rangle=\langle s\rangle \cong C_{2}$ and $\left\langle s^{\perp}\right\rangle=\langle r\rangle \cong C_{2}$, while $\left\langle t^{\perp}\right\rangle$ is trivial; and

- in $W_{3},\left\langle s_{i}^{\perp}\right\rangle=\left\langle s_{i-1}, s_{i+1}\right\rangle \cong D_{\infty}$ for each $1 \leq i \leq p$.

Lemma 2 Let $\mathcal{T}$ be a tree-wall of $X$ of type $s$. Let $\phi$ be a chamber which is epicormic at $\mathcal{T}$ and let $A$ be any apartment containing $\phi$.

(1) The intersection $\mathcal{T} \cap A$ is a wall of $A$, hence separates $A$.

(2) There is a bijection between the elements of the group $\left\langle s^{\perp}\right\rangle$ and the set of chambers of $A$ which are epicormic at $\mathcal{T}$ and in the same component of $A-\mathcal{T} \cap A$ as $\phi$.

Proof Part (1) is immediate from the definition of tree-wall. For Part (2), let $w \in\left\langle s^{\perp}\right\rangle$ and let $\psi=\psi_{w}$ be the unique chamber of $A$ such that $\delta_{W}(\phi, \psi)=w$. We claim that $\psi$ is epicormic at $\mathcal{T}$ and in the same component of $A-\mathcal{T} \cap A$ as $\phi$.

For this, let $s_{1} \cdots s_{n}$ be a reduced expression for $w$ and let $\alpha=\left(\phi_{0}, \ldots, \phi_{n}\right)$ be the minimal gallery from $\phi=\phi_{0}$ to $\psi=\phi_{n}$ of type $\left(s_{1}, \ldots, s_{n}\right)$. Since $w$ is in $\left\langle s^{\perp}\right\rangle$, we have $m_{s_{i} s}=2$ for $1 \leq i \leq n$. Hence by induction each $\phi_{i}$ is epicormic at $\mathcal{T}$, and so $\psi=\phi_{n}$ is epicormic at $\mathcal{T}$. Moreover, since none of the $s_{i}$ are equal to $s$, the gallery $\alpha$ does not cross $\mathcal{T}$. Thus $\psi=\psi_{w}$ is in the same component of $A-\mathcal{T} \cap A$ as $\phi$.

It follows that $w \mapsto \psi_{w}$ is a well-defined, injective map from $\left\langle s^{\perp}\right\rangle$ to the set of chambers of $A$ which are epicormic at $\mathcal{T}$ and in the same component of $A-\mathcal{T} \cap A$ as $\phi$. To complete the proof, we will show that this map is surjective. So let $\psi$ be a chamber of $A$ which is epicormic at $\mathcal{T}$ and in the same component of $A-\mathcal{T} \cap A$ as $\phi$, and let $w=\delta_{W}(\phi, \psi)$. 
If $\left\langle s^{\perp}\right\rangle$ is trivial then $\psi=\phi$ and $w=1$, and we are done. Next suppose that the chambers $\phi$ and $\psi$ are $t$-adjacent, for some $t \in S$. Since both $\phi$ and $\psi$ are epicormic at $\mathcal{T}$, either $t=s$ or $m_{s t}=2$. But $\psi$ is in the same component of $A-\mathcal{T} \cap A$ as $\phi$, so $t \neq s$, hence $w=t$ is in $\left\langle s^{\perp}\right\rangle$ as required. If $\left\langle s^{\perp}\right\rangle$ is finite, then finitely many applications of this argument will finish the proof. If $\left\langle s^{\perp}\right\rangle$ is infinite, we have established the base case of an induction on $n=l_{S}(w)$.

For the inductive step, let $s_{1} \cdots s_{n}$ be a reduced expression for $w$ and let $\alpha=$ $\left(\phi_{0}, \ldots, \phi_{n}\right)$ be the minimal gallery from $\phi=\phi_{0}$ to $\psi=\phi_{n}$ of type $\left(s_{1}, \ldots, s_{n}\right)$. Since $\phi$ and $\psi$ are in the same component of $A-\mathcal{T} \cap A$ and $\alpha$ is minimal, the gallery $\alpha$ does not cross $\mathcal{T}$. We claim that $s_{n}$ is in $s^{\perp}$. First note that $s_{n} \neq s$ since $\alpha$ does not cross $\mathcal{T}$ and $\psi=\phi_{n}$ is epicormic at $\mathcal{T}$. Now denote by $\mathcal{T}_{n}$ the tree-wall of $X$ containing the $s_{n}$-panel $\phi_{n-1} \cap \phi_{n}$. Since $\alpha$ is minimal and crosses $\mathcal{T}_{n}$, the chambers $\phi=\phi_{0}$ and $\psi=\phi_{n}$ are separated by the wall $\mathcal{T}_{n} \cap A$. Thus the $s$-panel of $\phi$ and the $s$-panel of $\psi$ are separated by $\mathcal{T}_{n} \cap A$. As the $s$-panels of both $\phi$ and $\psi$ are in the wall $\mathcal{T} \cap A$, it follows that the walls $\mathcal{T}_{n} \cap A$ and $\mathcal{T} \cap A$ intersect. Hence $m_{s_{n} s}=2$, as claimed.

Now let $w^{\prime}=w s_{n}=s_{1} \cdots s_{n-1}$ and let $\psi^{\prime}$ be the unique chamber of $A$ such that $\delta_{W}\left(\phi, \psi^{\prime}\right)=w^{\prime}$. Since $s_{n}$ is in $s^{\perp}$ and $\psi^{\prime}$ is $s_{n}$-adjacent to $\psi$, the chamber $\psi^{\prime}$ is epicormic at $\mathcal{T}$ and in the same component of $A-\mathcal{T} \cap A$ as $\phi$. Moreover $s_{1} \cdots s_{n-1}$ is a reduced expression for $w^{\prime}$, so $l_{S}\left(w^{\prime}\right)=n-1$. Hence by the inductive assumption, $w^{\prime}$ is in $\left\langle s^{\perp}\right\rangle$. Therefore $w=w^{\prime} s_{n}$ is in $\left\langle s^{\perp}\right\rangle$, which completes the proof.

Corollary 3 The following possibilities for tree-walls in $X$ may occur.

(1) Every tree-wall of type $s$ is reduced to a vertex if and only if $\left\langle s^{\perp}\right\rangle$ is trivial.

(2) Every tree-wall of type $s$ is finite but not reduced to a vertex if and only if $\left\langle s^{\perp}\right\rangle$ is finite but nontrivial.

(3) Every tree-wall of type $s$ is infinite if and only if $\left\langle s^{\perp}\right\rangle$ is infinite.

Proof Let $\mathcal{T}, \phi$, and $A$ be as in Lemma 2 above. The set of $s$-panels in the wall $\mathcal{T} \cap A$ is in bijection with the set of chambers of $A$ which are epicormic at $\mathcal{T}$ and in the same component of $A-\mathcal{T} \cap A$ as $\phi$.

For the examples in Section 1 above:

- in $X_{1}$, every tree-wall of type $s$ and of type $t$ is a vertex;

- in $X_{2}$, the tree-walls of types both $r$ and $s$ are finite and 1-dimensional, while every tree-wall of type $t$ is a vertex; and

- in $X_{3}$, all tree-walls are infinite, and are 1-dimensional. 
Corollary 4 Let $\mathcal{T}, \phi$, and $A$ be as in Lemma 2 above and let

$$
\rho=\rho_{\phi, A}: X \rightarrow A
$$

be the retraction onto $A$ centered at $\phi$. Then $\rho^{-1}(\mathcal{T} \cap A)=\mathcal{T}$.

Proof Let $\psi$ be any chamber of $A$ which is epicormic at $\mathcal{T}$ and is in the same component of $A-\mathcal{T} \cap A$ as $\phi$. Then by the proof of Lemma 2 above, $w:=\delta_{W}(\phi, \psi)$ is in $\left\langle s^{\perp}\right\rangle$. Let $\psi^{\prime}$ be a chamber in the preimage $\rho^{-1}(\psi)$ and let $A^{\prime}$ be an apartment containing both $\phi$ and $\psi^{\prime}$. Since the retraction $\rho$ preserves $W$-distances from $\phi$, we have that $\delta_{W}\left(\phi, \psi^{\prime}\right)=w$ is in $\left\langle s^{\perp}\right\rangle$. Again by the proof of Lemma 2, it follows that the chamber $\psi^{\prime}$ is epicormic at $\mathcal{T}$. But the image under $\rho$ of the $s$-panel of $\psi^{\prime}$ is the $s$-panel of $\psi$. Thus $\rho^{-1}(\mathcal{T} \cap A)=\mathcal{T}$, as required.

Lemma 5 Let $\mathcal{T}$ be a tree-wall and let $\phi$ and $\phi^{\prime}$ be two chambers of $X$. Let $\alpha$ be a minimal gallery from $\phi$ to $\phi^{\prime}$ and let $\beta$ be any gallery from $\phi$ to $\phi^{\prime}$. If $\alpha$ crosses $\mathcal{T}$ then $\beta$ crosses $\mathcal{T}$.

Proof Suppose that $\alpha$ crosses $\mathcal{T}$. Since $\alpha$ is minimal, there is an apartment $A$ of $X$ which contains $\alpha$, and hence the wall $\mathcal{T} \cap A$ separates $\phi$ from $\phi^{\prime}$. Choose a chamber $\phi_{0}$ of $A$ which is epicormic at $\mathcal{T}$ and consider the retraction $\rho=\rho_{\phi_{0}, A}$ onto $A$ centered at $\phi_{0}$. Since $\phi$ and $\phi^{\prime}$ are in $A, \rho$ fixes $\phi$ and $\phi^{\prime}$. Hence $\rho(\beta)$ is a gallery in $A$ from $\phi$ to $\phi^{\prime}$, and so $\rho(\beta)$ crosses $\mathcal{T} \cap A$. By Corollary 4 above, $\rho^{-1}(\mathcal{T} \cap A)=\mathcal{T}$. Therefore $\beta$ crosses $\mathcal{T}$.

Proposition 6 Let $\mathcal{T}$ be a tree-wall of type $s$. Then $\mathcal{T}$ separates $X$ into $q_{s}$ galleryconnected components.

Proof Fix an $s$-panel in $\mathcal{T}$ and let $\phi_{1}, \ldots, \phi_{q_{s}}$ be the $q_{s}$ chambers containing this panel. Then for all $1 \leq i<j \leq q_{s}$, the minimal gallery from $\phi_{i}$ to $\phi_{j}$ is just $\left(\phi_{i}, \phi_{j}\right)$, and hence crosses $\mathcal{T}$. Thus by Lemma 5 above, any gallery from $\phi_{i}$ to $\phi_{j}$ crosses $\mathcal{T}$. So the $q_{s}$ chambers $\phi_{1}, \ldots, \phi_{q_{s}}$ lie in $q_{s}$ distinct components of $X-\mathcal{T}$.

To complete the proof, we show that $\mathcal{T}$ separates $X$ into at most $q_{s}$ components. Let $\phi$ be any chamber of $X$. Then among the chambers $\phi_{1}, \ldots, \phi_{q_{s}}$, there is a unique chamber, say $\phi_{1}$, at minimal gallery distance from $\phi$. It suffices to show that $\phi$ and $\phi_{1}$ are in the same component of $X-\mathcal{T}$.

Let $\alpha$ be a minimal gallery from $\phi$ to $\phi_{1}$ and let $A$ be an apartment containing $\alpha$. Then there is a unique chamber of $A$ which is $s$-adjacent to $\phi_{1}$. Hence $A$ contains $\phi_{i}$ for some $i>1$, and the wall $\mathcal{T} \cap A$ separates $\phi_{1}$ from $\phi_{i}$. Since $\alpha$ is minimal and 
$d_{W}\left(\phi, \phi_{1}\right)<d_{W}\left(\phi, \phi_{i}\right)$, the Exchange Condition (see [5, page 35]) implies that a minimal gallery from $\phi$ to $\phi_{i}$ may be obtained by concatenating $\alpha$ with the gallery $\left(\phi_{1}, \phi_{i}\right)$. Since a minimal gallery can cross $\mathcal{T} \cap A$ at most once, $\alpha$ does not cross $\mathcal{T} \cap A$. Thus $\phi$ and $\phi_{1}$ are in the same component of $X-\mathcal{T}$, as required.

\section{Proof of Theorem}

Let $G$ be as in the introduction and let $\Gamma$ be a non-cocompact lattice in $G$ with strict fundamental domain. Fix a chamber $\phi_{0}$ of $X$. For each integer $n \geq 0$ define

$$
D(n):=\left\{\phi \in \operatorname{Ch}(X) \mid d_{W}\left(\phi, \Gamma \phi_{0}\right) \leq n\right\} .
$$

Then $D(0)=\Gamma \phi_{0}$, and for every $n>0$ every connected component of $D(n)$ contains a chamber in $\Gamma \phi_{0}$. To prove Theorem 1, we will show that there is no $n>0$ such that $D(n)$ is connected.

Let $Y$ be a strict fundamental domain for $\Gamma$ which contains $\phi_{0}$. For each chamber $\phi$ of $X$, denote by $\phi_{Y}$ the representative of $\phi$ in $Y$.

Lemma 7 Let $\phi$ and $\phi^{\prime}$ be $t$-adjacent chambers in $X$, for $t \in S$. Then either $\phi_{Y}=\phi_{Y}^{\prime}$, or $\phi_{Y}$ and $\phi_{Y}^{\prime}$ are $t$-adjacent.

Proof It suffices to show that the $t$-panel of $\phi_{Y}$ is the $t$-panel of $\phi_{Y}^{\prime}$. Since $Y$ is a subcomplex of $X$, the $t$-panel of $\phi_{Y}$ is contained in $Y$. By definition of a strict fundamental domain, there is exactly one representative in $Y$ of the $t$-panel of $\phi$. Hence the unique representative in $Y$ of the $t$-panel of $\phi$ is the $t$-panel of $\phi_{Y}$. Similarly, the unique representative in $Y$ of the $t$-panel of $\phi^{\prime}$ is the $t$-panel of $\phi_{Y}^{\prime}$. But $\phi$ and $\phi^{\prime}$ are $t$-adjacent, hence have the same $t$-panel, and so it follows that $\phi_{Y}$ and $\phi_{Y}^{\prime}$ have the same $t$-panel.

Corollary 8 The fundamental domain $Y$ is gallery-connected.

Lemma 9 For all $n>0$, the fundamental domain $Y$ contains a pair of adjacent chambers $\phi_{n}$ and $\phi_{n}^{\prime}$ such that, if $\mathcal{T}_{n}$ denotes the tree-wall separating $\phi_{n}$ from $\phi_{n}^{\prime}$ :

(1) the chambers $\phi_{0}$ and $\phi_{n}$ are in the same gallery-connected component of $Y-$ $\mathcal{T}_{n} \cap Y$;

(2) $\min \left\{d_{W}\left(\phi_{0}, \phi\right) \mid \phi \in \mathrm{Ch}(X)\right.$ is epicormic at $\left.\mathcal{T}_{n}\right\}>n$; and

(3) there is a $\gamma \in \operatorname{Stab}_{\Gamma}\left(\phi_{n}^{\prime}\right)$ which does not fix $\phi_{n}$.

Proof Fix $n>0$. Since $\Gamma$ is not cocompact, $Y$ is not compact. Thus there exists a tree-wall $\mathcal{T}_{n}$ with $\mathcal{T}_{n} \cap Y$ nonempty such that for every $\phi \in \mathrm{Ch}(X)$ which is epicormic at $\mathcal{T}_{n}, d_{W}\left(\phi_{0}, \phi\right)>n$. Let $s_{n}$ be the type of the tree-wall $\mathcal{T}_{n}$. Then by Corollary 8 
above, there is a chamber $\phi_{n}$ of $Y$ which is epicormic at $\mathcal{T}_{n}$ and in the same galleryconnected component of $Y-\mathcal{T}_{n} \cap Y$ as $\phi_{0}$, such that for some chamber $\phi_{n}^{\prime}$ which is $s_{n}$-adjacent to $\phi_{n}, \phi_{n}^{\prime}$ is also in $Y$. Now, as $\Gamma$ is a non-cocompact lattice, the orders of the $\Gamma$-stabilizers of the chambers in $Y$ are unbounded. Hence the tree-wall $\mathcal{T}_{n}$ and chambers $\phi_{n}$ and $\phi_{n}^{\prime}$ may be chosen so that $\left|\operatorname{Stab}_{\Gamma}\left(\phi_{n}\right)\right|<\left|\operatorname{Stab}_{\Gamma}\left(\phi_{n}^{\prime}\right)\right|$.

Let $\phi_{n}, \phi_{n}^{\prime}, \mathcal{T}_{n}$, and $\gamma$ be as in Lemma 9 above and let $s=s_{n}$ be the type of the tree-wall $\mathcal{T}_{n}$. Let $\alpha$ be a gallery in $Y-\mathcal{T}_{n} \cap Y$ from $\phi_{0}$ to $\phi_{n}$. The chambers $\phi_{n}$ and $\gamma \cdot \phi_{n}$ are in two distinct components of $X-\mathcal{T}_{n}$, since they both contain the $s$-panel $\phi_{n} \cap \phi_{n}^{\prime} \subseteq \mathcal{T}_{n}$, which is fixed by $\gamma$. Hence the galleries $\alpha$ and $\gamma \cdot \alpha$ are in two distinct components of $X-\mathcal{T}_{n}$, and so the chambers $\phi_{0}$ and $\gamma \cdot \phi_{0}$ are in two distinct components of $X-\mathcal{T}_{n}$. Denote by $X_{0}$ the component of $X-\mathcal{T}_{n}$ which contains $\phi_{0}$, and put $Y_{0}=Y \cap X_{0}$.

Lemma 10 Let $\phi$ be a chamber in $X_{0}$ that is epicormic at $\mathcal{T}_{n}$. Then $\phi_{Y}$ is in $Y_{0}$ and is epicormic at $\mathcal{T}_{n} \cap Y$.

Proof We consider three cases, corresponding to the possibilities for tree-walls in Corollary 3 above.

(1) If $\mathcal{T}_{n}$ is reduced to a vertex, there is only one chamber in $X_{0}$ which is epicormic at $\mathcal{T}_{n}$, namely $\phi_{n}$. Thus $\phi=\phi_{n}=\phi_{Y}$ and we are done.

(2) If $\mathcal{T}_{n}$ is finite but not reduced to a vertex, the result follows by finitely many applications of Lemma 7 above.

(3) If $\mathcal{T}_{n}$ is infinite, the result follows by induction, using Lemma 7 above, on

$$
k:=\min \left\{d_{W}(\phi, \psi) \mid \psi \text { is a chamber of } Y_{0} \text { epicormic at } \mathcal{T}_{n} \cap Y\right\} .
$$

Lemma 11 For all $n>0$, the complex $D(n)$ is not connected.

Proof Fix $n>0$, and let $\alpha$ be a gallery in $X$ between a chamber in $X_{0} \cap \Gamma \phi_{0}$ and some chamber $\phi$ in $X_{0}$ that is epicormic at $\mathcal{T}_{n}$. Let $m$ be the length of $\alpha$.

By Lemma 7 and Lemma 10 above, the gallery $\alpha$ projects to a gallery $\beta$ in $Y$ between $\phi_{0}$ and a chamber $\phi_{Y}$ that is epicormic at $\mathcal{T}_{n} \cap Y$. The gallery $\beta$ in $Y$ has length at most $m$.

It follows from (2) of Lemma 9 above that the gallery $\beta$ in $Y$ has length greater than $n$. Therefore $m>n$. Hence the gallery-connected component of $D(n)$ that contains $\phi_{0}$ is contained in $X_{0}$. As the chamber $\gamma \cdot \phi_{0}$ is not in $X_{0}$, it follows that the complex $D(n)$ is not connected.

This completes the proof, as $\Gamma$ is finitely generated if and only if $D(n)$ is connected for some $n$. 


\section{References}

[1] W Ballmann, J Świątkowski, On $L^{2}$-cohomology and property (T) for automorphism groups of polyhedral cell complexes, Geom. Funct. Anal. 7 (1997) 615-645 MR1465598

[2] H Bass, A Lubotzky, Tree lattices, Progress in Math. 176, Birkhäuser, Boston (2001) MR1794898 With appendices by Bass, L Carbone, Lubotzky, G Rosenberg and J Tits

[3] M Bourdon, Immeubles hyperboliques, dimension conforme et rigidité de Mostow, Geom. Funct. Anal. 7 (1997) 245-268 MR1445387

[4] L Carbone, H Garland, Existence of lattices in Kac-Moody groups over finite fields, Commun. Contemp. Math. 5 (2003) 813-867 MR2017720

[5] M W Davis, The geometry and topology of Coxeter groups, London Math. Soc. Monogr. Ser. 32, Princeton Univ. Press (2008) MR2360474

[6] J Dymara, T Januszkiewicz, Cohomology of buildings and their automorphism groups, Invent. Math. 150 (2002) 579-627 MR1946553

[7] R Gramlich, M Horn, B Mühlherr, Abstract involutions of algebraic groups and of Kac-Moody groups, to appear in J. Group Theory arXiv:0905.1280

[8] D A Kazhdan, On the connection of the dual space of a group with the structure of its closed subgroups, Funkcional. Anal. i Priložen. 1 (1967) 71-74 MR0209390

[9] A Kubena, A Thomas, Density of commensurators for uniform lattices of right-angled buildings arXiv:0812.2280

[10] MS Raghunathan, Discrete subgroups of algebraic groups over local fields of positive characteristics, Proc. Indian Acad. Sci. Math. Sci. 99 (1989) 127-146 MR1013536

[11] B Rémy, Construction de réseaux en théorie de Kac-Moody, C. R. Acad. Sci. Paris Sér. I Math. 329 (1999) 475-478 MR1715140

[12] A Thomas, Lattices acting on right-angled buildings, Algebr. Geom. Topol. 6 (2006) 1215-1238 MR2253444

[13] A Zuk, La propriété (T) de Kazhdan pour les groupes agissant sur les polyèdres, C. R. Acad. Sci. Paris Sér. I Math. 323 (1996) 453-458 MR1408975

School of Mathematics and Statistics, University of Sydney

Carslaw Building F07, Sydney NSW 2006, Australia

Department of Mathematics, University of Utah

155 South 1400 East, Room 233, Salt Lake City UT 84112-0090, USA

athomas@maths.usyd.edu.au, wortman@math.utah.edu

Received: 21 September $2010 \quad$ Revised: 6 January 2011 